\title{
Soziale Probleme, diskursive Praxis und das Problem mit der Kriminologie
}

\author{
Birgit Menzel · Jan Wehrheim
}

Eingegangen: 31. Mai 2020 / Angenommen: 21. September 2020 / Online publiziert: 27. Oktober 2020 (C) Der/die Autor(en) 2020

Zusammenfassung Der Aufsatz befasst sich mit Gemeinsamkeiten zwischen der Soziologie sozialer Probleme und der Kriminologie, insbesondere mit der Kontroverse um ätiologische und konstruktivistische Perspektiven auf soziale Probleme und abweichendes Verhalten. Am Beispiel aktueller Debatten über sexualisierte Gewalt werden die Defizite ätiologischer Analysen aufgezeigt. Der Beitrag kommt zu dem Schluss, dass die Soziologie sozialer Probleme ebenso wie die Kriminologie in einer Soziologie sozialer Problematisierungen und sozialer Kontrolle aufgeht.

\section{Social Problems, Discursive Practice, and the Problem with Criminology}

\begin{abstract}
The article focuses on similarities between sociology of social problems and criminology in general, on the controversy around etiological and constructivist perspectives on social problems and deviance in particular. Current debates on sexual violence show the deficiencies of etiological analyses. The paper concludes that sociology of social problems as well as criminology are dissolved in a sociology of social problematization and social control.
\end{abstract}

\footnotetext{
B. Menzel $(\bowtie)$

Fakultät Wirtschaft und Soziales, Department Public Management, HAW Hamburg, Berliner

Tor 5, 20099 Hamburg, Deutschland

E-Mail: birgit.menzel@haw-hamburg.de

J. Wehrheim

Institut für Soziale Arbeit und Sozialpolitik, Universität Duisburg-Essen,

Universitätsstraße 2, 45141 Essen, Deutschland

E-Mail: jan.wehrheim@uni-due.de
} 
Die Geschichte der Soziologie sozialer Probleme war und ist von der wissenschaftstheoretischen Kontroverse um konkurrierende Ansätze zum Verstehen und Erklären „sozialer Probleme“ gekennzeichnet. Auch diese Zeitschrift war von Beginn an durch sie geprägt, wie der (jenseits des Editorials) allererste Aufsatz, der vor 30 Jahren in dieser Zeitschrift erschien, zeigt: Günter Albrechts „Theorie sozialer Probleme im Widerstreit zwischen ,objektivistischen " und ,rekonstruktivistischen " Ansätzen“ (1990). Die Fortsetzung der Debatte zieht sich durch die Geschichte der Zeitschrift: 2001 erschien ein von Axel Groenemeyer herausgegebenes Heft mit dem Titel „Soziale Probleme - konstruktivistische Kontroversen und gesellschaftliche Herausforderungen“, 2006 aus Anlass des 30-jährigen Jubiläums der Sektion eines unter der Fragestellung „Die Soziologie sozialer Probleme in der Krise?“ (mit einer Fortsetzung in 2007), von vielen Einzelbeiträgen, die nicht in thematisch fokussierten Heften erschienen, einmal ganz abgesehen. Damals wie heute geht es im Kern darum, welche Fragen mit den als objektivistisch oder auch strukturfunktionalistisch bezeichneten Ansätzen im Anschluss vor allem an Robert K. Merton und den als interaktionistisch, konstruktivistisch, konstruktionistisch oder auch als rekonstruktionistisch benannten Zugängen im Anschluss unter anderem an Herbert Blumer beantwortet werden können. Außerdem dreht(e) sich die Kontroverse regelmäßig auch darum, ob sich beide Perspektiven zu etwas Drittem verbinden lassen.

Die Basisannahmen der beiden Ansätze sind schlicht erklärt (vgl. detailliert Peters 2002): Einmal wird davon ausgegangen, es gäbe eine bestimmbare Differenz zwischen einem Ist-Zustand eines sozialen (oder sozialstrukturellen) Phänomens und dem gesellschaftlich erwarteten Soll-Zustand. Die Aufgabe der Soziologie, so Merton (1975), sei es nicht nur, diese Diskrepanz zu beschreiben und zu analysieren, sondern auch, latente soziale Probleme zu identifizieren, um diese (sozial-)politisch bearbeiten zu können und die Gesellschaft zu ,verbessern“. Die im Folgenden als konstruktivistisch bezeichneten Ansätze hielten und halten dem entgegen, es sei nie möglich diese Diskrepanz und damit soziale Probleme objektiv zu erfassen. Bereits der Bezeichnung „Problem“ sei Normativität inhärent. Würde man Merton folgen, könnten nur die normativen Maßstäbe der jeweiligen Soziolog*innen das Kriterium zur Identifikation latenter Probleme sein. Als Konsequenz daraus bleibe lediglich und überzeugende Gegenargumente fehlen nach wie vor - den Prozess der Problematisierung, die „Karriere sozialer Probleme“ (Schetsche 2014), zu beschreiben und zu analysieren. Soziale Sachverhalte werden erst über die gesellschaftliche Definition und durch Problematisierungen und politische Auseinandersetzungen zu sozialen Problemen (vgl. auch Blumer 1975, S. 105f.).

Diese grundsätzliche Unterscheidung und Unvereinbarkeit der Ansätze lässt sich gleichermaßen auf die Kriminologie resp. die Kriminal- und Devianzsoziologie übertragen, auch wenn es immer wieder einmal Ansätze für eine einheitliche Theorie der Kriminalität gab (z. B. Hess und Scheerer 1997; Dollinger et al. 2014). „Kriminalität“ gilt üblicherweise als ein ,soziales Problem“, wobei dieses, so Albrecht (1990, S. 17), mangels „effektiver“ Behebung um das Problem „Furcht vor Kriminalität“" erweitert wurde. „Kriminalität“ weicht jedoch insofern von anderen problematisierten Phänomenen ab, als dass es erstens mit einer besonderen Etikettierung und Stigmatisierung einhergeht: Den eines Normverstoßes Beschuldigten wird vorge- 
worfen, dies absichtlich, schuldhaft, verantwortungsvoll oder auch bösartig getan zu haben. Zweitens ergibt sich ein gewisser Sonderstatus aufgrund der „Durchregulierung“ des „Problems“: Zu den Regelungen des Strafgesetzbuches oder der Strafprozessordnung kommen zahlreiche Institutionen, die zur Bearbeitung eines als vermeintlich objektiv vorliegenden „Problems“ geschaffen wurden. Durch ihre Existenz und Praxis bearbeiten sie das Problem und konstruieren es immer wieder neu mit: Polizei, Gericht, Jugendgerichtshilfe, Strafvollzug etc. pp. sind so gesehen Akteur*innen des Doing Social Problems (Groenemeyer 2010). Professions- oder systemtheoretisch argumentiert, sind sie genuin auf die Fortexistenz des „Problems“ angewiesen, ohne das ihre eigene Existenz in Frage gestellt würde. Mit welcher Vehemenz kontrollierende und besondernde Professionen und Institutionen auf Gefährdungen ihrer Existenz reagieren, lässt sich beispielhaft ablesen am Widerstand, den Sonderpädagogik und Sonderpädagog*innen gegen die 1973 vom Deutschen Bildungsrat verabschiedeten Empfehlungen zur Integration behinderter und von Behinderung bedrohter Kinder und Jugendlicher in das allgemeine Schulwesen leisteten (Eberwein 1998), ebenso an der Art und Weise, wie die grundstürzende Psychiatriekritik der 1970er-Jahre vom psychiatrischen System in einer ,,selbstbezüglichen Eigendynamik“ (von Kardorff 2017, S. 4) aufgesogen und im eigenen Interesse umdefiniert wurde.

Trotz gelegentlicher abolitionistischer Positionierungen zur Abschaffung von Gefängnissen und Strafrecht (vgl. etwa Papendorf 2016) waren Institutionen der Bearbeitung von „Kriminalität“ nie ernsthaft herausgefordert. Die Kriminologie als Wissenschaft von ,lawmaking, lawbreaking, and reactions to lawbreaking“ (Sutherland et al. 1992, S. 3) spaltet sich allerdings wie die Soziologie sozialer Probleme in zwei Perspektiven auf: einerseits ätiologische, positivistische Ansätze, die Kriminologie als exakte Wissenschaft begreifen und sich am erkenntnistheoretischen Erklärungsmodell bzw. am normativen Paradigma orientieren. Diese Kriminologie interessiert sich für die Ursachen und die Verbreitung krimineller Handlungen also für lawbreaking - und geht davon aus, diese von nicht-kriminellen Handlungen eindeutig und unabhängig von den Beobachter*innen unterscheiden zu können.

Daneben steht die oft als kritisch bezeichnete Kriminologie der Etikettierungsoder Definitionstheorie, die dem sozialwissenschaftlichen Verstehensmodell bzw. dem interpretativen Paradigma folgt. Sie stellt ,die gemeinsame Klassifizierung“ entsprechend etikettierter Personen und Handlungen „für analytische Zwecke“ (Goffman 1985, S. 180) in Frage. Dieser in der Kriminologie auch als Labeling Approach bezeichnete Ansatz fragt nach Prozessen der Normgenese sowie nach kontextabhängigen Zuschreibungen, in Folge derer soziales Handeln als kriminell be-deutet wird - also nach lawmaking und nach reactions to lawbreaking. Es sei erst die Bewertung einer Handlung, die diese kriminell werden lässt: ,Wir verurteilen sie [die Tat, d.A.] nicht, weil sie ein Verbrechen ist, sondern sie ist ein Verbrechen, weil wir sie verurteilen“, so Émile Durkheim (1988, S. 130), der jedoch kein Vertreter einer solchen konstruktivistisch orientierten Kriminologie war. Das Einzige, was strafbewehrte Handlungen, die eventuell als Schwarzfahren, Vergewaltigen, Steuerhinterziehung, Foltern, Taschendiebstahl, Vorbereiten eines Angriffskrieges, Versicherungsbetrug oder als Besitz von Cannabis definiert werden, gemeinsam haben, ist ihre mehr oder weniger häufige Etikettierung als Straftat. 
Kriminalität ist eine juristische und somit auch eine wertende Kategorie, an der sich die Soziologie nicht orientieren sollte, will sie nicht die herrschende Normativität übernehmen und sich nicht als eigenständige Disziplin selbst diskreditieren. Wir folgen darin Goffmans Ablehnung einer Devianzsoziologie, die ihren Gegenstand von der Gesellschaft übernimmt oder ihn sogar selbst schafft: „Gerade so, wie es iatrogene Störungen gibt, verursacht durch die Arbeit, die Mediziner leisten (was ihnen dann mehr zu tun gibt), so gibt es auch Personenkategorien, die von den Forschern der Gesellschaft erst geschaffen und dann von ihnen studiert werden“" (Goffman 1985, S. 172, FN 1). Ursachen, Verbreitung und Entwicklung von ,Kriminalität ‘ können keine sinnvollen Gegenstände soziologischer Analysen sein, diese müssen sich vielmehr auf Normen und Normalität richten - oder, um noch einmal Goffman zu zitieren: Es ist ,nicht das Andere ..., das man suchen sollte, um unsere Andersartigkeit zu verstehen, sondern das Gewöhnliche“ (Goffman 1985, S. 157). Gefragt werden kann dann auch, wieso bestimmte Handlungen/Konflikte unter bestimmten Bedingungen als Kriminalität gedeutet werden und in anderen Kontexten nicht, wie es dazu kommt, dass bestimmbare Handlungen per Gesetz kriminalisiert oder entkriminalisiert werden, sowie welche gesellschaftliche Funktion oder welche gesellschaftlichen Effekte (selektive) Kriminalisierungen haben. Dies führt zu den gemeinsamen Fragen der konstruktivistischen Soziologie sozialer Probleme und der definitionstheoretisch ausgerichteten Kriminologie resp. Kriminalsoziologie: Was sind die Bedingungen, wer sind die Akteur*innen der Problematisierung bzw. Kriminalisierung? Und was sagen uns die Erkenntnisse über Bedingungen und Akteur*innen über den gesellschaftlichen Status quo? Diese Fragen und die darauffolgenden Antworten führen weg von eigenen Problematisierungen und hin zu Erkenntnissen über die Produktion von Wissen, das von unterschiedlichen sozialen Akteur*innen genutzt wird (staatliche, soziale Bewegungen, privatwirtschaftliche ...). Auf diese Weise können auch Argumente für kritische, auf Veränderung der gesellschaftlichen Verhältnisse zielende Positionierungen geliefert werden.

Am Beispiel der Problematisierung sexueller Gewalt soll im Folgenden zwei bekannten Überlegungen zur Soziale-Probleme-Soziologie und zur Kriminologie nachgegangen und dabei angedeutet werden, wie kompliziert das Verhältnis von Wissensproduktion zu emanzipatorischen Bewegungen ist: zum einen eine methodologisch-theoretische Überlegung zu Wechselwirkungen zwischen Problemdiskursen und interaktiven Situationsbe-Deutungen, also dazu, was auch als diskursive Praxis bezeichnet werden kann, und damit verbunden zum anderen eine Überlegung zur aktiven oder passiven Rolle der Kriminologie und anderer Problem-Wissenschaften bei der Problemkonstruktion.

\section{Perspektiven auf Problemdiskurse und interaktive Situationsbe- Deutungen}

Sexuelle Beziehungen sind vielfältig reguliert. Was speziell reguliert ist und rechtlich und/oder moralisch besonders bewertet wird, variiert jedoch. Um es kompliziert zu formulieren: Als gewalttätig und als sexuell gedeutete, nicht einvernehmliche Kontakte zwischen Personen, die als männlich und weiblich kategorisiert werden, 
waren keineswegs immer von strafrechtlicher Relevanz (z.B. war Vergewaltigung bis 1997 in Deutschland ein ausschließlich außerehelicher Straftatbestand, der zudem ausschließlich Männer als Täter und ausschließlich Frauen als Opfer vorsah), ${ }^{1}$ wurden bei formaler Strafbarkeit eher selten so interpretiert (das Kontextmerkmal „kurzer Rock“ schützte männliche Beschuldigte oft vor der Zuschreibung von Täterschaft) und dementsprechend galten solche physischen Handlungen, unabhängig von der Häufigkeit ihres Auftretens, auch nicht als anerkanntes soziales Problem. Einvernehmliche sexuelle Beziehungen zwischen gleichgeschlechtlichen Personen, die von den Beteiligten selbst nicht als Gewalt definiert wurden, galten hingegen sehr wohl als soziales Problem und global gesehen hat sich in den letzten Jahrzehnten diesbezüglich relativ wenig verändert.

Betrachtet man nun neue Strafrechtsnormen sowohl als Indikator und Motor für Problematisierungen als auch als Bearbeitung und staatliche Anerkennung von sozialen Problemen, so zeigen sich verschiedene Strafverschärfungen, die jedoch ihrerseits spezifisch kontextualisiert sind und bei denen Wechselwirkungen zwischen Problemdiskursen und Situationsbe-Deutungen festzustellen sind. Im Konzept des Doing Social Problems wird davon ausgegangen, dass öffentliche, politische und durch kriminologische Forschungen und Positionierungen beeinflusste Diskurse über soziale Probleme „Problemerfahrungen strukturieren und ein kollektives Wissen bereitstellen, anhand dessen Menschen im Alltag und in Organisationen der Problembearbeitung ihr eigenes Denken, Fühlen und Handeln interpretieren und sie in Auseinandersetzung damit ihr Handeln ausrichten“ (Groenemeyer 2010, S. 24). Praxis wird damit nicht außerdiskursiv verortet, sondern Handeln, Be-Deutungen und Diskurse sind zusammen und in permanenten Wechselwirkungen zueinander zu denken. Die ätiologische Kriminologie versucht wiederum, unabhängig von solchen Überlegungen zu Reflexivität, inkriminierte Handlungen zu erklären, die dann in Problematisierungen wieder aufgegriffen werden. Kreislaufeffekte sind naheliegend. Entsprechende, theoretisch eigentlich banale, Wechselwirkungen gilt es in der Soziologie empirisch mit ihren variierenden Richtungen oder Dynamiken zu berücksichtigen, ohne jedoch dabei Kategorien nur zu reproduzieren. Zudem sollte eine zeitliche Komponente mitgedacht werden (zur methodologischen Diskussion der Verbindung von symbolischem Interaktionismus und Diskursanalyse in Anschluss an Adele Clark vgl. etwa Diaz-Bone 2012).

Exemplarisch empirisch veranschaulichen lassen sich solche Wechselwirkungen zwischen Diskursen und Situationsbe-Deutungen am Beispiel dessen, was medial und politisch als das „Kölner Silvester“ verhandelt wurde (zum [medialen] Diskurs im Nachgang der Ereignisse vgl. Werthschulte 2017; Drüeke 2016 sowie Dietze 2016a). Auch wenn eine „objektive“ Analyse dessen, was in der Silvesternacht 2015/2016 passierte, nicht möglich ist, lassen sich aus der polizeilichen Bearbeitung der Ereignisse ein paar Erkenntnisse gewinnen. In seinem Gutachten, das wegen des politischen Vorwurfs polizeilichen Versagens und der (später widerlegten) These

\footnotetext{
1 Vgl. auch die Kontroverse um das 1997er Abstimmungsverhalten des Kandidaten für den aktuellen CDU-Parteivorsitz Friedrich Merz: https://correctiv.org/faktencheck/politik/2018/11/14/dieseabgeordneten-stimmten-1997-gegen-die-strafbarkeit-von-vergewaltigung-in-der-ehe. Zugegriffen: 18. April 2020.
} 
organisierter sexueller Gewalt in Auftrag gegeben worden war, extrahierte Rudolf Egg aus 1580 Vorgängen 1022 auswertbare Fälle, von denen 302 als Sexualdelikte kategorisiert wurden (alle Zahlenangaben aus Egg 2016) ${ }^{2}$. Zwei Drittel der polizeilich aufgenommenen Fälle wurden hingegen ausschließlich oder zumindest auch als Eigentumsdelikte kategorisiert. Auffällig und an dieser Stelle von besonderem Interesse ist zudem zum einen, dass über $70 \%$ aller Anzeigen erst Tage bzw. Wochen später erfolgten (ebd., S. 34). Dies lässt aus definitionstheoretischer Perspektive den Schluss naheliegend erscheinen, dass die betroffenen Frauen erst aufgrund der medialen Thematisierung auf die Idee kamen, dass das Erlebte ggf. nicht nur ärgerlich, erniedrigend, abstoßend und verängstigend gewesen war, sondern zudem justiziabel - oder auch, dass sie erst aufgrund des medialen Diskurses annahmen, ihre Anzeige würde überhaupt ernst genommen. In der eigentlich keineswegs definitionstheoretisch geprägten gutachterlichen Stellungnahme dokumentiert Egg zum anderen, dass es bei den Anzeigen unterschiedliche Definitionen und auch nachträgliche Umdefinitionen durch die Polizist*innen, die die Anzeige aufnahmen, gegeben hat, die sich jeweils auf das Sexualstrafrecht bezogen:

Dabei zeigte sich eine sehr uneinheitliche Verwendung der jeweiligen Zuordnung durch die Polizei, d.h. es wurden Tatvorwürfe, die nach der inhaltlichen Beschreibung in den Anzeigen sehr ähnlich waren, einmal als Beleidigung ( 185), ein anderes Mal als - der formal schwerere Straftatbestand - sexuelle Nötigung/Vergewaltigung ( $\$ 177$ ) bezeichnet. In etlichen Fällen wurde diesbezüglich eine zunächst vorgenommene Zuordnung später (meist handschriftlich) „,nach oben“ oder auch (seltener) „unten““ abgeändert. (Egg 2016, S. 10) ${ }^{3}$

Auch in Bezug auf die polizeilichen Einordnungen erscheint es insofern naheliegend, dass der nachfolgende Diskurs Situationsdeutungen ex post beeinflusst hat.

Vergleicht man Schilderungen der Ereignisse in der Kölner Nacht mit Berichten zum jährlichen Münchner Oktoberfest, wie sie etwa in der Süddeutschen Zeitung dokumentiert wurden - ,Security und Polizei greifen nur ein, wenn einer ausrastet und etwa mit einem Maßkrug auf einen Kontrahenten einprügeln will. Der Griff an eine Pobacke als Grund? Da gäb es ja viel zu tun“; „Betrunkene entblößen sich, fassen Frauen unters Dirndl und feuern sich gegenseitig an"4 allem eine Erkenntnis der interaktionistischen (Kriminal-)Soziologie naheliegend: Die Be-Deutung der Dinge variiert mit den Kontexten, innerhalb derer die Dinge

\footnotetext{
${ }^{2}$ Egg, Rudolph. 2016. Gutachterliche Stellungnahme zu den anonymisierten Strafanzeigen der Ermittlungsgruppe Neujahr. Wiesbaden (unveröffentlicht).

${ }^{3}$ Egg, Rudolph. 2016. Gutachterliche Stellungnahme zu den anonymisierten Strafanzeigen der Ermittlungsgruppe Neujahr. Wiesbaden (unveröffentlicht).

${ }^{4} \mathrm{https}: / /$ www.sueddeutsche.de/muenchen/sexuelle-uebergriffe-auf-dem-oktoberfest-entbloessung-imbierzelt-1.1151859. Zugegriffen: 18. April 2020. Vgl. auch: Ursprünglicher Wortlaut eines Wiesn-Reports der Polizei-München aus dem September 2015, also kurz vor dem „Kölner Silvester“: „Ein spaßig gemeinter Griff unter den Rock seiner amerikanischen Wiesn-Bekanntschaft endete für einen 20-jährigen Deggendorfer äußerst schmerzhaft. Die ,Rockbesitzerin' drehte sich mitsamt Maßkrug um und das Trinkgefäß landete wuchtig auf dem Kopf des kecken Burschen." Der Einsatz des Maßkruges führte zur Anzeige wegen gefährlicher Körperverletzung: https://www.stern.de/panorama/weltgeschehen/oktoberfest--wiesich-ein-polizeibericht-anhoert--wenn-deutsche-maenner-frauen-bedraengen-6641130.html. Zugegriffen: 18. April 2020.
} 
wahrgenommen werden. Naheliegend ist dabei, dass, neben der ätiologischen Überlegung, der Griff an den Körper könne eine erfolgversprechende Ablenkung gewesen sein, um sich etwa Smartphones anzueignen, die entscheidenden, definitionsleitenden Kontextmerkmale die Unterschiede bei der Sprache, Haut- und Haarfarbe der vermeintlichen Täter waren. Im Zuge rassistischer Diskurse über Migration, wie sie verstärkt ab 2015 wieder auftraten, wurden physikalisch identische Handlungen offenbar unterschiedlich be-deutet.

Eine Verschärfung des Sexualstrafrechts folgte 2016 u. a. dahingehend, dass der $\S$ 177 StGB um den Tatbestand ,sexuellen Übergriff“" erweitert wurde, da die meisten der im Nachgang des „Kölner Silvester“ inkriminierten Handlungen bis dato nicht strafrechtlich relevant gewesen sein dürften. Der „Nein heißt nein“-Grundsatz wurde 2016 gerade auch von ,a-typischen“ Moralunternehmer*innen (vgl. Scheerer 1986) betont, d.h. von durchaus unterschiedlichen Strömungen sozialer, feministischer Bewegungen. Zeitgleich wurde das Aufenthaltsrecht geändert - erweiterte Abschiebegründe, die von Teilen dieser Unternehmer*innen jedoch strikt, aber erfolglos abgelehnt wurden, ebenso wie das polizeiliche Racial Profiling bei den Silvesterfeiern ein Jahr später in Köln. ${ }^{5}$ Das soziale Problem „,sexualisierte Gewalt gegen Frauen" erfuhr eine erweiterte rechtliche Anerkennung mit einer spezifischen Konnotation, die ohne nationalistisch-rassistische Diskurse vermutlich nicht so leicht durchsetzbar gewesen wäre. Gabriele Dietze (2016b) spricht in diesem Zusammenhang von Ethnosexismus. Das soziale Problem hätte insofern auch als „,sexualisierte Gewalt gegen deutsche Frauen“ oder „,durch ausländische Männer“ konstruiert werden können. So formuliert, wäre die staatliche Anerkennung 2016 allerdings wohl noch ausgeblieben.

Auffällig im politisch-wissenschaftlichen Diskurs (nicht nur) um sexualisierte Gewalt, wie er nach diesem Silvester auch im Zusammenhang mit dem so genannten \#metoo-Diskurs geführt wurde, ist die theoretisch-methodologische Nähe unterschiedlicher, sich oft als kritisch begreifender Wissenschaften (z.B. Genderstudies, Rassismusforschung, Kriminologie), welche jedoch die Erkenntnisse der konstruktivistischen Soziologie immer dann ausklammern, wenn es nicht um den eigenen, präferierten Forschungsgegenstand oder die eigene politische Programmatik bzw. normative Positionierung geht. So kritisieren etwa Daniela Klimke und Rüdiger Lautmann (2018) das Schweigen der Kritischen Kriminologie beim verstärkt ab 2017 auftretenden \#metoo-Diskurs. Sie klagen, die punitiven und freiheitseinschränkenden Effekte des Diskurses würden ignoriert und argumentieren:

Dass man diesen Extremfall punitiver Entwicklungen überwiegend nicht instanzenkritisch bewertet und die schwächeren AkteurInnen nicht als AdressatInnen, sondern aufseiten der Strafrechtsverschärfungen wähnt, zeigt das Problem auf, das die Kritische Kriminologie mit den ,atypischen Moralunternehmern" (Scheerer 1986) und ihren TrittbrettfahrerInnen hat, soweit diese imstande sind, sich als Opfer vorzuführen. Den feministischen Anliegen hat sich längst eine medial höchst erfolgreiche, schrille Skandalisierungsoffensi-

\footnotetext{
5 Vgl. z.B. https://ausnahmslos.org/; https://www.gwi-boell.de/de/2017/01/25/postkoelnialismus. Zugegriffen: 18. April 2020.
} 
ve um sexuelle Gewalt bemächtigt, was sich aktuell an der Kampagne „Me too" und zuvor an etlichen, nicht ganz so spektakulären Problematisierungswellen zeigt. Die Kritischen KriminologInnen lassen sich hiervon zwar nicht aktiv einnehmen, aber sie verstummen. (...) Die Missbrauchsdiskurse um Sexualgewalt stellen vollkommen bösartige Täter gegen gute unschuldige Opfer. Damit produzieren sie eine vorreflexive Einigkeit in der Diagnose eines moralischen Verfalls. Wir alle werden, sei es real, sei es symbolisch, zu Opfern der sexuellen Aggression gegen die Schuldlosen. (...) Die von bestimmten BewegungssprecherInnen vorgegebenen Muster wurden nahtlos übernommen und damit popularisiert: die Rede von der Großen Zahl, die Dramatisierung über spektakuläre Dunkelziffern und deren Versinnbildlichung (,,alle drei Minuten wird ..."), die Nivellierung der Begrifflichkeit, die Metaphern wie Trauma und Mord an Seelen, die Täter-Opfer-Dichotomie. Der Gewaltbegriff wird dabei weit über seine strafrechtliche Definition hinaus ausgedehnt und fasst eine Vielzahl verdächtiger Situationen in der Klammer eines strukturellen Sexismus zusammen. Dominanz vs. Hilflosigkeit werden zu den vorrangigen Deutungsmustern sozialer Interaktionen, wodurch sich die viktimistisch-punitive Perspektive ausbreitet und in endloser Folge immer neue Sexualskandale produziert. Gegen alle Abwägungen und Differenzierungen zeigt sich dieser Diskurs immun. (Klimke und Lautmann 2018, S. 26f.)

Die Kritische Kriminologie wird hier also wegen ihrer Passivität kritisiert. Damit wird ihr indirekt eine Beteiligung an Dramatisierungen und Kriminalisierungen vorgeworfen, da sie innerhalb des Diskurses keine Gegenposition stärke. Sie schweigt halt. ${ }^{6}$ Erwägungen eines „,strukturellen Sexismus“ werden in der Perspektive von Klimke und Lautmann hingegen nicht näher thematisiert. Gesellschaftlich strukturelle Machtverhältnisse im Kontext von Geschlecht und Sexualität, patriarchale Herrschaft werden in dieser Perspektive nicht problematisiert. Die Fragen sind andere. Die Problematisierung bezieht sich auf die Moral und die strafrechtliche Bearbeitung mit allem zusätzlichen Leid, das sie produziert, und zwar vor dem empirischen Hintergrund vielfältiger Kriminalisierungen von vielen Varianten sexueller Aktivitäten und Beziehungen und nicht zuletzt der Verfolgung und Ermordung vieler entsprechend Beschuldigter.

Intersektional orientierte Wissenschaftler*innen und Aktivist*innen hingegen greifen zwar die vielfachen Verbindungen und Wirkungen des Komplexes Rassismus/Sexismus, strukturelle Machtverhältnisse und der darin enthaltenen Wissensbestände in ihren Analysen (und Problematisierungen) auf, die machtsensible und konstruktivistische Perspektive endet jedoch bei der verdinglichten „Kriminalität“. Intendierte und nicht-intendierte Effekte des Strafrechts und seiner Anwendung bleiben ebenso unhinterfragt wie die Täter-Opfer-Dichotomie bestehen bleibt und implizit davon ausgegangen wird, Handlungen seien objektiv bestimmbar und bessere Videoüberwachung während der Kölner Silvesternacht hätte mehr Licht ins Dunkel gebracht (vgl. Dietze 2016a, S. 93; Drüeke 2016). Die Gegenstände der

\footnotetext{
${ }^{6}$ Wobei es in der deutschsprachigen Kritischen Kriminologie auch nicht so viele Wissenschaftler*innen gibt, die in den Bereichen forschen und sich überhaupt kompetent positionieren könnten.
} 
Problematisierung bzw. die zugrundeliegenden sozialen Handlungen werden als unabhängig von interaktiven Be-Deutungen gegeben angesehen und nicht hinterfragt. Die Annahme also, dass Frauen die Handlungen nur oder vor allem deswegen als sexualisiert und/oder gewalttätig gedeutet haben könnten und nicht als Normalität oder normalisiertes Ärgernis wie auf dem Oktoberfest, weil die Handelnden nicht weiß-deutsch aussahen und/oder weil genau diese Konstellation medial problematisiert wurde, wird ausgeklammert. Auf einer klassischen ätiologischen Basis wird die normative Bewertung - Kriminalität/sexuelle Gewalt - zum objektiven sozialen Sachverhalt, auf dem aufruhend dann nur noch um die ,richtige“ Konstruktion des sozialen Problems im öffentlichen Diskurs gerungen wird: Sexismus und Gewalt gegen Frauen an sich oder sexuelle Gewalt gegen Frauen durch junge, eventuell muslimische Männer. Ähnliches gilt auch für die \#metoo-Debatte anhand der Beispiele möglicher Handlungen prominenter Filmproduzenten, Schauspieler und Regisseure: Handlungen werden - vergleichbar mit Howard S. Beckers „objektivistische[m] Rest“ (Keckeisen 1974, S. 42) - als bislang nur noch nicht entdeckte/ problematisierte Regelverstöße objektiviert und Situationsbe-Deutungen als über die Jahrzehnte und unabhängig von den Akteur*innen und öffentlichen Diskursen stabil und objektiv angenommen. Auch die Erkenntnis, dass die Vergangenheit vor dem Hintergrund des aktuellen Wissens interpretiert wird, bleibt dabei unberücksichtigt.

Diskurse führen dazu, situiertes Handeln ggf. anders zu deuten als dies bei anderen, früheren rechtlichen und diskursiven Rahmungen der Fall war. Normen stellen zudem keine Handlungsanleitungen dar, sondern bieten als sprachliche Konstrukte nur Orientierungshilfe bei der Interpretation von Situationen - und umgekehrt. Sie müssen in die Situationen hineingeholt werden und in den Situationen wird interaktiv ausgehandelt, welches Handeln von wem als situationsadäquat angesehen wird - und zwar zunächst unabhängig von institutionellen Perspektiven und Strafrecht.

Mit Michel Foucault argumentiert, sind die strafrechtlich und diskursiv ausgetragenen Konflikte unter anderem im Zuge der \#metoo-Debatte um Sexualität und Gewalt mit Blick auf Subjekt und Gesellschaft vor allem eins: durch und durch ambivalent. Diese Ambivalenz lässt sich nicht auflösen und sie ist auch individuell höchst relevant, denn mögliches persönliches Leiden hängt mit der gesellschaftlichen Bewertung des Erlebten zusammen. Einerseits werden in den Beispielen Machtverhältnisse neu ausgehandelt und im Feld der Sexualität Jahrhunderte patriarchaler Herrschaft hinterfragt. Andererseits und gleichzeitig werden über diese konflikthaften Aushandlungen Vorstellungen von Moral transportiert und neue Normen verhandelt, die Beziehungen zwischen Personen neu rahmen. Dietze (2016a, S. 100) argumentiert, ,dass das spätmoderne Sexualitätsdispositiv auf der okzidentalen Seite nicht mehr als ein repressiv imaginiertes Verbotssystem zu verstehen ist, sondern als Imperativ der Gewährung und Freiheit funktioniert“. Dies scheint dann widersprüchlich, wenn sexuelle Freiheit symbolisch über das Strafrecht gewährleistet bzw. eingeschränkt werden soll. Beim law in action (Pound 1910) ist zudem anzunehmen, dass dies nicht nur geschlechtsspezifisch, sondern auch in Bezug auf Herkunfts- oder Kulturzuschreibung der Beteiligten sowie deren sozialen Status selektiv handelt und Strafrecht als Ressource Machtverhältnisse selektiv verändert: Im „Köln-Diskurs“ wäre es die Unterscheidung zwischen als deutsch und als nichtdeutsch klassifizierten Männern; im \#metoo-Diskurs liegt der Fokus auf Konstella- 
tionen, in denen Status- und damit Machtdifferenzen besonders groß sind oder so erscheinen.

\section{Kriminologie und Soziologie als Akteurinnen der Problemkonstruktion und als kritische Wissenschaften}

Kriminologie und Kriminalsoziologie beeinflussen entsprechende Diskurse, sie sind an der Veränderung von (Strafrechts-)Normen beteiligt und dadurch beeinflussen sie auch deren situationsspezifische Interpretationen - auch wenn der Einfluss meist als eher gering einzuschätzen ist bzw. nur dann wirksam wird, wenn Erkenntnisse an interessierte und machtvolle Positionen anschlussfähig sind.

Die Kriminologie kann, wie erwähnt, nicht „Kriminalität“ in Ursachen, Regelmäßigkeiten und Umgang damit untersuchen, wenn sie nicht die Definitionen und Perspektiven der Justiz resp. von Instanzen sozialer Kontrolle übernehmen will. Die ätiologische Kriminologie und die Soziologie abweichenden Verhaltens sind insofern eigentlich genauso wissenschaftlich obsolet wie die objektivistische/ strukturfunktionalistische Soziologie sozialer Probleme: Sie haben keinen eigenen Gegenstand, sondern schaffen sich diesen im Anschluss an vorgegebene normative und juristische Kategorien selbst. Je nachdem welche Fragen Kriminolog*innen und Soziolog*innen stellen, welchen Paradigmen sie folgen, welche Forschungen sie betreiben und welche Kategorisierungen sie übernehmen und fortschreiben oder auch, ob und wann sie Fragen und Forschungen nicht verfolgen, sind sie und damit ihre Disziplinen aktive oder passive Akteur*innen der sozialen Problematisierung. Was die Soziologie aber neben der nüchternen Beschreibung und Analyse von Handlungen, die manchmal als Kriminalität bezeichnet werden, kann, ist Definitionsund Konstruktionsprozesse zu beschreiben und zu analysieren - und auch, ob daran kollektive Deutungsprozesse anschließen, die „Kriminalität“ anschließend zu einem sozialen Problem werden lassen und vice versa. Übrigbleiben kann also eigentlich nur eine „Soziologie sozialer Kontrolle“, die die Produktion von Normen und Normalitäten in den Blick nimmt, und/oder eine ,Soziologie sozialer Problematisierung“. Denn: sowohl „Abweichung/Kriminalität“ als auch ,soziale Probleme“ sind letztendlich Ergebnisse von diskursiver Praxis sozialer Kontrolle.

Allerdings ist Wissenschaft bekanntlich selbst Teil von Gesellschaft und hegemonialen Diskursen und Forschung folgt auch im weitesten Sinne ökonomischen Rationalitäten, was manche Forschungen und Positionierungen erschwert. Das „Problem der Ambivalenz" ausklammernd, vermuten Klimke und Lautmann Risiken für das wissenschaftliche Renommee als Erklärung für die (aus ihrer Perspektive politisch problematischen) Nicht-Positionierungen zu ,heiklen Themen“:

Als erstes steht hier wohl die Erwägung an, ob das eigene Renommee beschädigt wird, wenn man sich auf einem belohnungsarmen Feld engagiert. Kriminalität an sich zählt ja bereits dazu, insofern man nicht beim Täter-Fangenund-unschädlich-Machen mittut (und selbst dann noch bleiben Strafrecht und Kriminologie prestigeschwache Fächer, akademisch wie beruflich). Selbstdemontage droht, wenn jemand sich der Brandung einer erregten öffentlichen 
Meinung entgegenstellt. AmtsinhaberInnen tun durchaus Recht daran, den Kredit ihrer Einrichtung zu schützen; denn davon hängen Projekte und Stellen ab. Rücksicht auf die Reputation darf prinzipiell als rational gelten. Märtyrertum nützt einer Sache meist erst postmortal. (Klimke und Lautmann 2018, S. 29f)

Die Schwierigkeiten der Kritischen Kriminologie und der konstruktivistischen Soziologie sozialer Probleme reichen aber noch weiter (vgl. zur Kritik an der Sicherheitsforschung: Wehrheim 2018). So sind bestimmte Themen und Perspektiven anschlussfähiger als andere: einerseits in Bezug auf hegemoniale Lehrinhalte (von der Denomination von Professuren, über Studiengänge bis zu Modulhandbüchern) und der damit verbundenen Nachfrage auf den Arbeitsmärkten für Kriminolog*innen und Vertreter*innen anderer, ,devianznaher“ Disziplinen und Professionen (z.B. Sozialarbeit). Ethnomethodologie, Interaktionismus oder machtsensible und herrschaftskritische Punitivitätsverweigerung sind schwerer vermittelbar als alltagstheoretisch anschlussfähige Inhalte und Theorien und sie werden außerhalb der Wissenschaft in Landeskriminalämtern oder Jugendgerichtshilfen nicht nachgefragt. Andererseits sind Themen und Perspektiven unterschiedlich anschlussfähig in Bezug auf die Frage danach, welche Forschung überhaupt finanziert wird und damit auch, wie wissenschaftlicher Nachwuchs sich finanzieren kann. Sich hegemonialen Forschungsthemen und -perspektiven zu verweigern, bedeutet eben häufig auch, Arbeit nicht bezahlt zu bekommen, und Wissenschaft ist jenseits von Idealen von Berufung, Freiheit und Unabhängigkeit meist schlicht Erwerbsarbeit. Kriminolog*innen haben wie Vertreter*innen anderer Problemprofessionen auch ein Interesse am Erhalt und der Rekonstruktion ihres Gegenstandes - auch wenn die Reflexion des eigenen Tuns selten intentional und derart kalkulierend verweigert werden dürfte. Die Konsequenzen zu ziehen, kann ähnlich riskant und kostspielig sein wie sich ins Märtyrertum zu stürzen.

Sieht man das Strafrecht als Herrschaftsinstrument an, mit dem Konflikte enteignet werden (Christie 1977), und das dadurch auch dann nicht geeignet ist - im hier gewählten Beispiel - die patriarchale Strukturierung von Gesellschaft aufzulösen, wenn Machtverhältnisse formal als law in the books (Pound 1910) verändert werden, so muss der Blick auf genau solche Funktionen der Problematisierung und Kriminalisierung gerichtet werden. Daran kann eine Soziologie sozialer Kontrolle und eine Soziologie sozialer Problematisierung anknüpfen. Strafrecht als Gegenstand der Kriminologie und oft Endpunkt von Karrieren sozialer Probleme sollte dahingehend thematisiert werden, inwiefern es als Instrument der Individualisierung von „Ärgernissen und Lebenskatastrophen“ (Hanak et al. 1989) dient und, paradoxerweise, die Konfliktbeteiligten gleichzeitig ihrer Deutungshoheit beraubt sowie soziale Ungleichheit und Hierarchien legitimiert. Als Soziologe klagte Heinz Steinert 1981 noch vor Gründung dieser Zeitschrift, dass er den Begriff „,soziale Probleme“ nicht mehr hören könne:

„Probleme“ ist einerseits eine Verharmlosung der Absurditäten, Unmöglichkeiten und Grausamkeiten, denen Gesellschaftsformationen ihre Angehörigen aussetzen (man denke etwa an Kriege, Hungersnöte, langdauernde Arbeitslosigkeit, regelmäßige und massenhafte Verkehrstote oder industrielle Vergiftung 
ganzer Landstriche), und andererseits eine Dramatisierung der geringfügigen Schwierigkeiten und Ärgernisse, die Mitglieder von Gesellschaften anderen bereiten und die ebenfalls unter diesem Stichwort abgehandelt werden (man denke etwa an Homosexualität, voreheliche Liebschaften, Erziehungsschwierigkeiten oder Eigenwilligkeiten der Haartracht). Und das Beiwort „Soziale“ impliziert, es handle sich um „Probleme“ der Gesellschaft (nicht darum, dass jemand oder etwas einem anderen ein „Problem“ bereitet), was wiederum einerseits eine Verharmlosung ist (weil es die Kämpfe wegeskamotiert, um die es dabei geht, wenn etwas zu einem „Sozialen Problem“ erklärt wird oder nicht und wenn es dann in einer bestimmten Weise angegangen wird oder nicht) und andererseits eine Dramatisierung (indem es so tut, als wäre alles, was irgendwen stört, gleich eine Gefährdung des gesamten Bestands der bestehenden Gesellschaft und ihrer Funktionsweise). Der Begriff ist damit in erster Linie nicht analytisch, sondern moralisch und politisch (oder unmoralisch und unpolitisch). (Steinert 1981, S. 56)

Die mit diesen Beispielen beschriebene Kritik am Begriff als moralisch und politisch und als unmoralisch und unpolitisch ist eigentlich ebenfalls eine moralische und politische. Für eine wissenschaftliche Perspektive aber wird an dieser Stelle gleichwohl Robert K. Merton mit seiner Frage nach manifesten und vor allem auch nach „latenten Funktionen“ wieder überraschend anschlussfähig, wenn, wie bei Steinert, die makrostrukturellen Funktionen und Effekte sowie die damit verbundenen Hintergründe und Interessen der Akteur*innen in den Blick genommen werden: allerdings nicht von ,sozialen Problemen“, sondern von Problematisierungen. Dies verlangt, gegenwärtige Gesellschaften und soziale Ordnungen nicht einfach als gesetzt und „gut“ zu betrachten. Das heißt auch: An dieser Stelle können Soziologie und Kriminologie als Soziologie sozialer Kontrolle resp. sozialer Problematisierung über entsprechende Fragestellungen und die genuine wissenschaftliche Aufgabe des Hinterfragens der Dinge zu einer kritischen Wissenschaft werden, die damit auch Wissen für Veränderungen bemängelter gesellschaftlicher Verhältnisse produziert. Als handlungs- und gesellschaftstheoretische Soziologie geht es dann darum, Verhältnisse, Prozesse und Beziehungen zu analysieren. Freiheit bedeutet zunächst einmal, die Bedingungen und Strukturen der Unfreiheit zu erkennen. Über die Produktion solchen Wissens wird Wissenschaft anschlussfähig für Kritik an diesen Bedingungen und Strukturen. Nicht einfach „Herrschaftswissen“ (Steinert 1998) zu (re-)produzieren und sich nicht die Fragen und Kategorien für die Forschung von Instanzen sozialer Kontrolle vorgeben zu lassen, sondern reflexiv mit diesen, dem eigenen Denken, Forschen und Lehren umzugehen, ist nicht leicht, aber die Forderung ist deshalb nicht falsch und sie gilt ganz besonders für die Kriminologie.

Funding Open Access funding enabled and organized by Projekt DEAL.

Open Access Dieser Artikel wird unter der Creative Commons Namensnennung 4.0 International Lizenz veröffentlicht, welche die Nutzung, Vervielfältigung, Bearbeitung, Verbreitung und Wiedergabe in jeglichem Medium und Format erlaubt, sofern Sie den/die ursprünglichen Autor(en) und die Quelle ordnungsgemäß nennen, einen Link zur Creative Commons Lizenz beifügen und angeben, ob Änderungen vorgenommen wurden. 
Die in diesem Artikel enthaltenen Bilder und sonstiges Drittmaterial unterliegen ebenfalls der genannten Creative Commons Lizenz, sofern sich aus der Abbildungslegende nichts anderes ergibt. Sofern das betreffende Material nicht unter der genannten Creative Commons Lizenz steht und die betreffende Handlung nicht nach gesetzlichen Vorschriften erlaubt ist, ist für die oben aufgeführten Weiterverwendungen des Materials die Einwilligung des jeweiligen Rechteinhabers einzuholen.

Weitere Details zur Lizenz entnehmen Sie bitte der Lizenzinformation auf http://creativecommons.org/ licenses/by/4.0/deed.de.

\section{Literatur}

Albrecht, Günter. 1990. Theorie sozialer Probleme im Widerstreit zwischen „objektivistischen“ und „rekonstruktionistischen“ Ansätzen. Soziale Probleme 1:5-20.

Blumer, Herbert. 1975. Soziale Probleme als kollektives Verhalten. In Menschliche Bedürfnisse und soziale Steuerung, Hrsg. Karl-Otto Hondrich, 102-113. Reinbek: Rowohlt.

Christie, Nils. 1977. Conflicts as property. British Journal of Criminology 17:1-15.

Diaz-Bone, Rainer. 2012. Review Essay: Situationsanalyse - Strauss meets Foucault? [21 Absätze]. Forum Qualitative Sozialforschung / Forum: Qualitative Social Research, 14(1), Art. 11. http://nbnresolving.de/urn:nbn:de:0114-fqs1301115. Zugegriffen: 18. Apr. 2020.

Dietze, Gabriele. 2016a. Das „Ereignis Köln“. Femina Politica 1:93-102.

Dietze, Gabriele. 2016b. Sex-Mob-Narrative um die Kölner Sylvesternacht. movements. Journal for Critical Migration and Border Regime Studies 2:177-185.

Dollinger, Bernd, Matthias Rudolph, Henning Schmidt-Semisch, und Monika Urban. 2014. Konturen einer allgemeinen Theorie der Kriminalität als kulturelle Praxis (ATKAP). Poststrukturalistische Perspektiven. Kriminologisches Journal 46:67-88.

Drüeke, Ricarda. 2016. Die TV-Berichterstattung in ARD und ZDF über die Silvesternacht 2015/16 in Köln. Studie im Auftrag des Gunda-Werner-Instituts für Feminismus und Geschlechterdemokratie der Heinrich-Böll-Stiftung. https://heimatkunde.boell.de/sites/default/files/http__www.gwi-boell. de_sites_default_files_web_161122_e-paper_gwi_medienanalysekoeln_v100.pdf. Zugegriffen: 18. Apr. 2020.

Durkheim, Émile. 1988. Über soziale Arbeitsteilung. Frankfurt a.M.: Suhrkamp.

Eberwein, Hans. 1998. Ein Rückblick nach 25 Jahren Integrationsentwicklung - Die Empfehlungen des Deutschen Bildungsrates ,Zur pädagogischen Förderung behinderter und von Behinderung bedrohter Kinder und Jugendlicher“ von 1973. Gemeinsam leben - Zeitschrift für integrative Erziehung 6:61-64.

Goffman, Erving. 1985. Stigma. Über Techniken der Bewältigung beschädigter Identität. Frankfurt a.M.: Suhrkamp.

Groenemeyer, Axel. 2010. Doing Social Problems - Doing Social Control. Mikroanalysen der Konstruktion sozialer Probleme in institutionellen Kontexten. Ein Forschungsprogramm. In Doing Social Problems. Mikroanalysen der Konstruktion sozialer Probleme und sozialer Kontrolle in institutionellen Kontexten, Hrsg. Axel Groenemeyer, 13-56. Wiesbaden: Springer VS.

Hanak, Gerhard, Johannes Stehr, und Heinz Steinert. 1989. Ärgernisse und Lebenskatastrophen. Über den alltäglichen Umgang mit Kriminalität. Bielefeld: AJZ.

Hess, Henner, und Sebastian Scheerer. 1997. Was ist Kriminalität? Skizze einer konstruktivistischen Kriminalitätstheorie. Kriminologisches Journal 29:83-155.

von Kardorff, Ernst. 2017. 40 Jahre gesellschaftspolitisches Projekt „Psychiatriereform“ - Was ist daraus geworden? soziale psychiatrie 41:4-8.

Keckeisen, Wolfgang. 1974. Die gesellschaftliche Definition abweichenden Verhaltens. Perspektiven und Grenzen des labeling approach. Weinheim: Juventa.

Klimke, Daniela, und Rüdiger Lautmann. 2018. Das Schweigen der Kritischen Kriminologie. Kriminologisches Journal 50:25-33.

Merton, Robert K. 1975. Soziologische Diagnose sozialer Probleme. In Menschliche Bedürfnisse und soziale Steuerung, Hrsg. Karl-Otto Hondrich, 113-129. Reinbek: Rowohlt.

Papendorf, Knut. 2016. Überlegungen zum Abolitionismus heute. Kriminologisches Journal 48:169-183.

Peters, Helge. 2002. Soziale Probleme und soziale Kontrolle. Wiesbaden: Westdeutscher Verlag.

Pound, Roscoe. 1910. Law in books and law in action. American Law Review 44:12-36. 
Scheerer, Sebastian. 1986. Atypische Moralunternehmer. Kriminologisches Journal, Bd. 1. Beiheft, 133-155. Weinheim: Juventa.

Schetsche, Michael. 2014. Empirische Analyse sozialer Probleme. Wiesbaden: Springer VS.

Steinert, Heinz. 1981. Widersprüche, Kapitalstrategien und Widerstand oder: Warum ich den Begriff „Soziale Probleme" nicht mehr hören kann. Versuch eines theoretischen Rahmens für die Analyse der politischen Ökonomie sozialer Bewegungen und „Sozialer Probleme“. Kriminalsoziologische Bibliografie 8:56-89.

Steinert, Heinz. 1998. Die organisierte Verhinderung von Wissen. In Kein Staat zu machen. Zur Kritik der Sozialwissenschaften, Hrsg. Christoph Görg, Roland Roth, 291-312. Münster: Westfälisches Dampfboot.

Sutherland, Edwin H., Donald R. Cressey, und David F. Luckenbill. 1992. Principles of criminology. New York: General Hall.

Wehrheim, Jan. 2018. Kritik der Versicherheitlichung. Thesen zur (sozialwissenschaftlichen) Sicherheitsforschung. Kriminologisches Journal 50:211-221.

Werthschulte, Christian. 2017. „Nach“ Köln ist wie „vor“ Köln. Die Silvesternacht und ihre Folgen. Bundeszentrale für politische Bildung. https://www.bpb.de/apuz/239696/die-silvesternacht-und-ihrefolgen. Zugegriffen: 18. Apr. 2020. 